\title{
PARTITIONS OF THE PLANE INTO SETS HAVING POSITIVE MEASURE IN EVERY NON-NULL MEASURABLE PRODUCT SET
}

BY

\author{
PAUL ERDŐS AND JOHN C. OXTOBY
}

1. Introduction. The following question was posed by D. Maharam: Can one divide the unit square into two or more measurable sets each of which has a non-null intersection with every product set $A \times B$ of positive measure, where $A$ and $B$ are subsets of the unit interval?

In this paper we construct a class of such partitions of the plane, including some that retain the property under various transformations. Not all known partitions are included in this class; in particular it does, not include one found by Maharam and A. H. Stone (unpublished), the first example which appears to have been considered. As side results we obtain a generalization of a theorem of Steinhaus, and a theorem on extension of a measure-preserving homeomorphism from a plane Cantor set. The property of being a member of a partition is shown to be nonexceptional in the sense of category in the space of measurable sets. We also consider the problem of partitioning a general product space.

If $X$ and $Y$ are measure spaces, we shall say that a set $E \subset X \times Y$ has property (M) relative to a set $U \subset X \times Y$ if $\mu(E \cap(A \times B))>0$ whenever $\mu(A \times B)>0$ and $A \times B \subset U$. Here $\mu$ denotes the independent product measure. A partition $\left\{E_{i}\right\}$ of $U$ will be called an (M)-partition of $U$ if each set $E_{i}$ has property (M) relative to $U$. Such a partition is necessarily countable if $U$ contains a product set of positive finite measure. A subset $E$ of a measure space with a topology is said to be metrically dense if $\mu(E \cap U)>0$ for every nonempty open set $U$.

It may be remarked that a measurable subset of the plane is metrically dense if and only if every equivalent set is topologically dense, and that it has property (M) relative to the plane if and only if it is metrically dense on every measurable product set that is metrically dense in itself.

It is easy to construct a partition of the line or plane into a sequence of metrically dense sets. It suffices to enumerate a countable base $\left\{U_{j}\right\}$, and construct a double sequence of disjoint nowhere dense sets $C_{i j}$ with $C_{i j} \subset U_{j}$ and $m\left(C_{i j}\right)>0$. Then the sets $D_{i}=U_{j} C_{i j}$ constitute such a partition, when the complement of their union is adjoined to one of the sets.

It is also easy to partition the plane into measurable sets (even uncountably many null sets) each of which has a nonempty intersection with every non-null measurable product set. As Helson [2] has observed, following Steinhaus, any union of straight lines of slope one has this property if it meets

Received by the editors June 30, 1954. 
the $x$-axis in a dense set. This follows from a theorem of Steinhaus [8], according to which, if $A$ and $B$ are linear sets of positive measure, the set of differences $\{x-y: x \in A, y \in B\}$ contains an interval. Pushing this idea a step further, we shall show that a union of lines of slope one has a non-null intersection with every non-null measurable product set if it meets the $x$-axis in a metrically dense set.

Theorem 1. A measurable set of the form $E=\{(x, y): x-y \in D\}$ has property (M) relative to the plane if and only if $D$ is metrically dense on the line.

Proof. If $A$ and $B$ are linear sets of finite positive measure, then

$$
\begin{aligned}
\mu(E \cap(A \times B)) & =\iint \chi_{D}(x-y) \chi_{A}(x) \chi_{B}(y) d x d y \\
& =\iint \chi_{D}(u) \chi_{A}(u+v) \chi_{B}(v) d u d v=\int_{D} f(u) d u,
\end{aligned}
$$

where $f(u)=\int \chi_{A}(u+v) \chi_{B}(v) d v=m(A \cap(u+B))$. The function $f$ is non-negative and continuous $\left[9\right.$, p. 50]; in fact $f(-u)$ is the convolution $\chi_{-A} * \chi_{B}$ of two functions belonging to $L_{2}$. Moreover, the above calculation with $D=X$ shows that $\int f(u) d u=\mu(A \times B)>0$. Hence $f$ is positive on some interval, and therefore $\int_{D} f(u) d u>0$ if $D$ is metrically dense on the line. The necessity of the condition is obvious.

Thus if $\left\{D_{i}\right\}$ is a partition of the line into metrically dense sets, the sets $E_{i}=\left\{(x, y): x-y \in D_{i}\right\}$ constitute an (M)-partition of the plane. However, these sets $E_{i}$ no longer have property (M) when the plane is referred to axes making an angle of $45^{\circ}$ with the original ones. This suggests the further problem, likewise posed by Maharam and A. H. Stone, of partitioning the plane into sets that have property $(M)$ when the plane is referred to axes in any orientation. More generally, if $T$ is any specified class of transformations with domain and range in the plane, we shall say that $\left\{E_{i}\right\}$ is a $T$-invariant (M)partition of the plane if $\left\{t E_{i}\right\}$ is an (M)-partition of the range of $t$, for each $t \in T$. It should be noted that any (M)-partition of the plane is invariant under the group of translations of the plane.

2. A generalization of Steinhaus' theorem. To facilitate the construction of invariant partitions, we generalize Theorem 1 as follows.

THEOREM 2. If $u(x, y)$ is a real-valued function of class $C^{\prime}$ on an open set $U$ in the plane, and if $u_{x} \neq 0$ and $u_{y} \neq 0$ almost everywhere in $U$, then any set of the form $E=\{(x, y): u(x, y) \in D\}$, where $D$ is metrically dense on the line, has the property that $\mu(E \cap(A \times B))>0$ whenever $\mu(U \cap(A \times B))>0$.

Proof. The open set where $u_{x} \neq 0$ and $u_{y} \neq 0$ can be represented as a countable union of closed rectangles. For given $A$ and $B$ with $\mu(U \cap(A \times B))>0$, at least one of these rectangles has a non-null intersection with $A \times B$. It 
therefore suffices to prove the theorem under the assumption that $A \times B$ is contained in a closed rectangle $I \times J$, on which $\left|u_{x}\right|$ and $\left|u_{y}\right|$ lie between positive bounds, say $1 / \lambda$ and $\lambda$. Let $u(x, y)$ be redefined outside $I \times J$ so that these bounds hold throughout the plane, and so that the extended function remains of class $C^{\prime}$. Then the equations $u=u(x, y), v=y$, define a 1:1 mapping of class $C^{\prime}$ of the $(x, y)$-plane onto the $(u, v)$-plane. The inverse transformation $x=x(u, v), y=v$, is likewise of class $C^{\prime}$, and its Jacobian $1 / u_{x}$ is nowhere zero. We have

$$
\begin{aligned}
\mu(E \cap(A \times B)) & =\iint \chi_{D}(u(x, y)) \chi_{A}(x) \chi_{B}(y) d x d y \\
& =\iint \chi_{D}(u) \chi_{A}(x(u, v)) \chi_{B}(v) \frac{1}{\left|u_{x}\right|} d u d v \\
& \geqq \frac{1}{\lambda} \iint \chi_{D}(u) \chi_{A}(x(u, v)) \chi_{B}(v) d u d v \\
& =\frac{1}{\lambda} \int_{D} f(u) d u,
\end{aligned}
$$

where

$$
f(u)=\int \chi_{A}(x(u, v)) \chi_{B}(v) d r .
$$

As before, it will suffice to show that $f$ is continuous and not identically zero. The latter property follows from the estimation:

$$
\begin{aligned}
\int f(u) d u & \geqq \frac{1}{\lambda} \iint \chi_{A}(x(u, v)) \chi_{B}(v) \frac{1}{\left|u_{x}\right|} d u d v \\
& =\frac{1}{\lambda} \iint \chi_{A}(x) \chi_{B}(y) d x d y \\
& =\frac{1}{\lambda} \mu(A \times B)>0 .
\end{aligned}
$$

The function $f(u)$ is a kind of generalized convolution, whose continuity can be proved in the same way as that of an ordinary convolution. In fact, let $\epsilon>0$ be arbitrary and let $g(x)$ and $h(y)$ be continuous functions that vanish outside $I$ and $J$, are bounded by 1 , and satisfy

$$
\int\left|\chi_{A}(x)-g(x)\right| d x<\epsilon, \quad \int\left|\chi_{B}(y)-h(y)\right| d y<\epsilon .
$$

Put $f_{0}(u)=\int g(x(u, v)) h(v) d v$; then $f_{0}$ is continuous for all $u$. On any line $u=$ constant, $d v / d x=-u_{x} / u_{y}$; hence 


$$
\begin{aligned}
& \left|f(u)-f_{0}(u)\right| \leqq \int\left|\chi_{A}(x(u, v))-g(x(u, v))\right| \chi_{B}(v) d v \\
& +\int|g(x(u, v))|\left|\chi_{B}(v)-h(v)\right| d v \\
& \leqq \int\left|\chi_{A}(x)-g(x)\right|\left|\frac{u_{x}}{u_{y}}\right| d x+\int\left|\chi_{B}(v)-h(v)\right| d v \\
& <\lambda^{2} \epsilon+\epsilon \text {. }
\end{aligned}
$$

It follows that $f$ is continuous for all $u$.

Theorem 2 implies the following generalization of Steinhaus' theorem.

Theorem 3. If $A$ and $B$ are linear sets of positive measure, and if $u(x, y)$ is a function of class $C^{\prime}$ on an open set $U$ with $\mu(U \cap(A \times B))>0$, and if $u_{x} \neq 0$ and $u_{y} \neq 0$ almost everywhere in $U$, then the set $\{u(x, y): x \in A, y \in B\}$ contains an interval.

Proof. Suppose the contrary for some pair of sets $A$ and $B$ and function $u(x, y)$. We may assume that $A$ and $B$ are bounded and closed, in which case the set $\{u(x, y): x \in A, y \in B\}$ is closed and nowhere dense, since it is supposed to contain no interval. The complement $D$ of this linear set is then metrically dense on the line, and the set $E=\{(x, y): u(x, y) \in D\}$ is disjoint to $A \times B$, contrary to Theorem 2.

3. Invariant partitions. To construct a partition invariant relative to a given class $T$ of $C^{\prime}$-transformations, it is sufficient (in view of Theorem 2) to find a function $u(x, y)$ which after transformation by an arbitrary member of $T$ has partial derivatives a.e. different from zero. Accordingly, we seek a function whose level lines transform into a family of curves with tangents a.e. parallel to neither axis.

For example, let $T$ be the group of affine transformations, and take $u=\arctan (y / x)$. The transformed function $u=\operatorname{arc} \tan ((c x+d y+f) /(a x$ $+b y+e)), a d-b c \neq 0$, has $u_{x} \neq 0$ and $u_{y} \neq 0$ except on at most three straight lines. It follows that any partition of the plane into sets $E_{i}$ each of which is a union of rays from the origin through a set metrically dense on the unit circle is an (M)-partition which retains the property under all affine transformations. In particular, such sets $E_{i}$ have property (M) when the plane is referred to axes in any orientation.

That an (M)-partition can be invariant under a very large class of transformations will now be shown.

THEOREM 4. Let $f$ be a continuous function on $-\infty<x<+\infty$ with the property that no function of class $C^{\prime}$ is equal to $f$ on a set of positive measure (an example is given below), and let $F(x)=\int_{0}^{x} f d x$. Let $\left\{D_{i}\right\}$ be a partition of the line into metrically dense sets. Then the sets $E_{i}=\left\{(x, y): y-F(x) \in D_{i}\right\}$ con- 
stitute an (M)-partition of the plane T-invariant under the class $T$ of all 1:1 transformations of class $C^{\prime \prime}$ with open domain and range in the plane and nowhere vanishing Jacobian.

Proof. By hypothesis, $F$ is of class $C^{\prime}$ on $(-\infty,+\infty)$, and if $G$ is any function of class $C^{\prime \prime}$ on an interval then $G^{\prime}(x) \neq F^{\prime}(x)$ a.e. Let $t$ be an arbitrary member of $T$, and let $t^{-1}$ be defined on its domain $U$ by the equations $\xi=\xi(x, y)$, $\eta=\eta(x, y)$. Then $t E_{i}$ is a set of the type considered in Theorem 2, with $u(x, y)=\eta(x, y)-F(\xi(x, y))$. This function is obviously of class $C^{\prime}$; hence it only remains to show that $u_{x} \neq 0$ and $u_{y} \neq 0$ a.e. in $U$.

Suppose $u_{x}=0$ on a set of positive plane measure. Then some line $y=y_{0}$ meets this set in a set of positive linear measure. That is, $\eta_{x}\left(x, y_{0}\right)$ $-F^{\prime}\left(\xi\left(x, y_{0}\right)\right) \xi_{x}\left(x, y_{0}\right)=0$ on a linear set $A$ of positive measure. Let $x_{0} \in A$ be a point where $A$ has density 1 . Then $\xi_{x}\left(x_{0}, y_{0}\right) \neq 0$, since otherwise the Jacobian of $t^{-1}$ would vanish at $\left(x_{0}, y_{0}\right)$. Hence $\xi_{x}\left(x, y_{0}\right) \neq 0$ in an interval about $x_{0}$, and the equations $\xi=\xi\left(x, y_{0}\right), \eta=\eta\left(x, y_{0}\right)$ define implicitly a function $\eta=G(\xi)$ of class $C^{\prime \prime}$ in an interval $I$ about $\xi_{0}=\xi\left(x_{0}, y_{0}\right)$, with $G^{\prime}(\xi)=\eta_{x}\left(x, y_{0}\right) / \xi_{x}\left(x, y_{0}\right)$. Thus $G^{\prime}(\xi)=F^{\prime}(\xi)$ on the set $B=I \cap\left\{\xi\left(x, y_{0}\right): x \in A\right\}$, which has density 1 at $\xi_{0}$. Hence $G^{\prime}(\xi)=F^{\prime}(\xi)$ on a set of positive measure, a contradiction. Similarly, $u_{y} \neq 0$ a.e. in $U$.

A function $f(x)$ with the required properties can be defined as follows. Let $\phi(x)$ be the distance from $x$ to the nearest integer, and let $a_{n}=2^{n(3 n-1) / 2}$ $(n \geqq 0)$. Then $\phi_{n}(x)=2 \phi\left(a_{n} x / 2\right)$ is a piecewise linear continuous function made up of linear segments with slope $\pm a_{n}$ and range $[0,1]$. Define

$$
f(x)=\sum_{n=1}^{\infty} \phi_{n}(x) / a_{n-1} \text {. }
$$

It is clear that $f$ is continuous on $(-\infty,+\infty)$. Moreover, for any $x$ and $n$ the points $\left(x+h, \phi_{n}(x+h)\right)$ and $\left(x, \phi_{n}(x)\right)$ lie on the same linear segment of the graph of $\phi_{n}$ for all $h$ in either $1 / 4 a_{n}<h<1 / 2 a_{n}$ or $-1 / 2 a_{n}<h<-1 / 4 a_{n}$. We shall show that for any such value of $h$ the contribution of $\phi_{n}$ to the difference quotient of $f$ is dominant. In fact,

$$
\left|\frac{f(x+h)-f(x)}{h}\right| \geqq \frac{a_{n}}{a_{n-1}}-\sum_{0<k<n} \frac{a_{k}}{a_{k-1}}-\sum_{k>n} \frac{4 a_{n}}{a_{k-1}} .
$$

Since $a_{k} / a_{k-1}=2^{3 k-2}$ and $a_{n} / a_{n+k} \leqq 2^{-4 k}$ for $k>0$, a simple calculation shows that

$$
\sum_{0<k<n} \frac{a_{k}}{a_{k-1}}<\frac{1}{7} \frac{a_{n}}{a_{n-1}} \text { and } \sum_{k>n} \frac{4 a_{n}}{a_{k-1}}<\frac{64}{15} .
$$

Consequently, for any value of $x$ and $n$, the inequality 


$$
\left|\frac{f(x+h)-f(x)}{h}\right| \geqq \frac{6}{7} 2^{3 n-2}-\frac{64}{15}
$$

holds for all $h$ in an interval of length $1 / 4 a_{n}$ contained in $\left(x-1 / 2 a_{n}\right.$, $\left.x+1 / 2 a_{n}\right)$. It follows that if $g(x)=f(x)$ on a set $A$ of positive measure, and if $x_{0} \in A$ is a point where $A$ has density 1 , then at least one of the derived numbers of $g(x)$ at $x=x_{0}$ is infinite. Consequently if $g$ is differentiable in an interval, then $g(x) \neq f(x)$ a.e. in the interval.

We now consider another application of Theorem 2 to the construction of an invariant partition.

TheOREM 5. Let $F(x)$ be a function of class $C^{\infty}$ on $(-\infty,+\infty)$ whose Taylor series at every point has zero radius of convergence. Let $\left\{D_{i}\right\}$ be a partition of the line into metrically dense sets. Then the sets $E_{i}=\left\{(x, y): y-F(x) \in D_{i}\right\}$ constitute an (M)-partition of the plane which is $T$-invariant under the class $T$ of all 1:1 real analytic, nonsingular transformations with domain and range in the plane.

Proof. Consider any $t \in T$ and let $t^{-1}$ be defined in its domain $U$ by $\xi=\xi(x, y), \eta=\eta(x, y)$. As before, let $u(x, y)=\eta(x, y)-F(\xi(x, y))$. Then $u$ is of class $C^{\infty}$ in $U$ and, by Theorem 2 , to show that $t E_{i}$ has property (M) relative to $U$ it will suffice to show that $u_{x} \neq 0$ and $u_{y} \neq 0$ a.e. in $U$. In fact, we shall show that on any line $y=y_{0}$ the equation $u_{x}=0$ can hold only at isolated points (a difference between the present proof and that of Theorem 4). For suppose the contrary; then for some point $\left(x_{0}, y_{0}\right)$ in $U$ and some sequence $\left\{x_{n}\right\}$ with limit $x_{0}, x_{n} \neq x_{0}$ and $u_{x}\left(x_{n}, y_{0}\right)=0$. Hence $\eta_{x}\left(x_{n}, y_{0}\right)-F^{\prime}\left(\xi\left(x_{n}, y_{0}\right)\right)$ $\cdot \xi_{x}\left(x_{n}, y_{0}\right)=0$ for $n \geqq 0$. It follows that $\xi_{x}\left(x_{0}, y_{0}\right) \neq 0$, since the Jacobian of $t^{-1}$ does not vanish at $\left(x_{0}, y_{0}\right)$. By continuity, $\xi_{x}(x, y)$ has constant sign throughout a neighborhood of $\left(x_{0}, y_{0}\right)$, which we may assume contains all the points $\left(x_{n}, y_{0}\right)$. Hence the sequence $\xi_{n}=\xi\left(x_{n}, y_{0}\right)$ converges to $\xi_{0}, \xi_{n} \neq \xi_{0}$, and $F^{\prime}\left(\xi_{n}\right)$ $=\eta_{x}\left(x_{n}, y_{0}\right) / \xi_{x}\left(x_{n}, y_{0}\right)$ for all $n \geqq 0$. Also, the equations $\eta=\eta\left(x, y_{0}\right), \xi=\xi\left(x, y_{0}\right)$ define a real analytic function $\eta=G(\xi)$ in an interval about $\xi_{0}$, and $G^{\prime}(\xi)$ $=\eta_{x}\left(x, y_{0}\right) / \xi_{x}\left(x, y_{0}\right)$. Hence $G^{\prime}\left(\xi_{n}\right)=F^{\prime}\left(\xi_{n}\right)$ for all $n$. Because $F$ and $G$ are of class $C^{\infty}$ at $\xi_{0}$, and $\xi_{0}$ is a point of accumulation of $\left\{\xi_{n}\right\}$, it follows that all derivatives of $F(\xi)-G(\xi)$ vanish at $\xi_{0}$. Hence the Taylor series of $F$ and $G$ at $\xi_{0}$ differ by a constant; a contradiction, since the Taylor series of $G$ has positive radius of convergence and that of $F$ does not. Similarly, on any line $x=x_{0}$ the equation $u_{y}=0$ can hold only at isolated points.

An example of a function $F(x)$ with the required properties is

$$
F(x)=\sum_{i=1}^{\infty} n_{i}^{-2 i} \cos n_{i} x, \quad \text { where } \quad n_{i}=2^{4^{i}} .
$$

Note first, by induction, that for this sequence $\left\{n_{i}\right\}$, 


$$
n_{k}>n_{k-1} \geqq 8, \quad \frac{1}{4} n_{k} \geqq 1+\sum_{i=1}^{k-1} n_{i}^{2 k+1-2 i}, \quad \text { and } \quad \frac{1}{4} n_{k}>2^{3^{k}} \text {, }
$$

for all integers $k>1$. That $F(x)$ is of class $C^{\infty}$ on $(-\infty,+\infty)$ is clear. It remains to show that for each value of $x_{0}$ the sequence $a_{n}=\left(\left|F^{(n)}\left(x_{0}\right)\right| / n !\right)^{1 / n}$ is unbounded. We shall estimate $a_{2 k+1}$ or $a_{2 k+2}$ according as $\left|\sin n_{k} x_{0}\right| \geqq 1 / 2$ or $\left|\cos n_{k} x_{0}\right| \geqq 1 / 2$. For each $k$ at least one of these inequalities holds, and the following computations show that the corresponding derived series is dominated by its $k$ th term.

If $\left|\sin n_{k} x_{0}\right| \geqq 1 / 2$ then

$$
\begin{aligned}
\left|F^{(2 k+1)}\left(x_{0}\right)\right| & =\left|\sum_{i=1}^{\infty} n_{i}^{2 k+1-2 i} \sin n_{i} x_{0}\right| \\
& \geqq \frac{1}{2} n_{k}-\sum_{i=1}^{k-1} n_{i}^{2 k+1-2 i}-\sum_{i=1}^{\infty} 8^{1-2 i} \\
& \geqq \frac{1}{4} n_{k} \geqq 2^{3^{k}} .
\end{aligned}
$$

If $\left|\cos n_{k} x_{0}\right| \geqq 1 / 2$ then

$$
\begin{aligned}
\left|F^{(2 k+2)}\left(x_{0}\right)\right| & =\left|\sum_{i=1}^{\infty} n_{i}^{2 k+2-2 i} \cos n_{i} x_{0}\right| \\
& \geqq \frac{1}{2} n_{k}^{2}-n_{k}\left(1+\sum_{i=1}^{k-1} n_{i}^{2 k+1-2 i}\right) \geqq \frac{1}{4} n_{k} \geqq 2^{3^{k}} .
\end{aligned}
$$

Hence for any $x_{0}$ and $k>1$ there exists an integer $n>k(n=2 k+1$ or $2 k+2)$ such that $a_{n}>\left(2^{3^{k} / 2 k+2}\right) / 2 k+2$. It follows that $\lim \sup a_{n}=\infty$.

4. The impossibility of certain invariant (M)-partitions. Having found an (M)-partition invariant under as large a class as that of all 1:1 nonsingular transformations of class $C^{\prime \prime}$, it is natural to ask what upper bounds can be assigned to $T$. It is easy to see that if $T$ is the class of all $1: 1$ measure-preserving transformations, then no invariant (M)-partition is possible, because any set $E$ of positive finite plane measure can be mapped onto a square by such a transformation. It is also easy to see that no (M)-partition can be invariant under all homeomorphisms. Somewhat deeper is the fact that no (M)-partition can retain the property under all area-preserving automorphisms of the plane. This is a consequence of the following theorem.

Theorem 6. For any plane set $E$ with $\mu(E)>\alpha>0$ there exists an areapreserving automorphism $h$ of the plane such that $h(E)$ contains a product set of measure $\alpha$. Moreover, $h$ can be taken equal to the identity outside some rectangle $R$.

Proof. Choose $R$ so that $\mu(E \cap R)>\alpha$, and let $P$ be a perfect, 0 -dimensional 
subset of $E \cap R$, interior to $R$, metrically dense in itself, and such that $\mu(P)$ $>\alpha$. Let $P_{0}$ be a countable dense subset of $P$, and let $N_{1}=P-P_{0}$. Then $N_{1}$ is the image of the set $N$ of irrational numbers in $(0,1)$ by a homeomorphism $f_{1}$ [3, vol. I, p. 349]. Moreover, $N_{1}$ is metrically dense in itself and $\mu\left(N_{1}\right)$ $=\mu(P)$. Let $N_{2}$ be the set of all points with irrational coordinates in a rectangle of area $\mu(P)$ concentric with $R$. Let $f_{2}$ be a homeomorphism of $N$ onto $N_{2}$. Then the Borel measures defined in $I=[0,1]$ by $m_{1}(A)=\mu\left(f_{1}(A)\right)$ and $m_{2}(A)=\mu\left(f_{2}(A)\right)$ correspond under the automorphism $f$ of $I$ defined by the equation $m_{1}([0, f(x)])=m_{2}([0, x])$; that is, $m_{1}(f(A))=m_{2}(A)$ for all Borel sets $A$. The transformation $f_{1} f f_{2}^{-1}$ is therefore an area-preserving homeomorphism of $N_{2}-D_{2}$ onto $N_{1}-D_{1}$, where $D_{2}$ and $D_{1}$ are the countable sets $D_{2}=f_{2} f^{-1}\left(R_{0}\right), D_{1}=f_{1} f\left(R_{0}\right)$, and $R_{0}$ is the set of rational numbers in $I$. Let $C_{1}$ and $C_{2}$ be linear Cantor sets such that $C_{1} \times C_{2} \subset N_{2}-D_{2}$ and $\mu\left(C_{1} \times C_{2}\right)=\alpha$. Let $K_{2}=C_{1} \times C_{2}$ and $K_{1}=f_{1} f f_{2}^{-1}\left(K_{2}\right)$. Then $h_{0}=f_{2} f^{-1} f_{1}^{-1}$ is an area-preserving homeomorphism of $K_{1}$ onto $K_{2}$. Since $K_{1} \subset E$ and $K_{2}$ is a product set of measure $\alpha$ the proof of Theorem 6 is completed by the following lemma.

Lemma A. If $h_{0}$ is an area-preserving homeomorphism of $K_{1}$ onto $K_{2}$, where $K_{1}$ and $K_{2}$ are plane Cantor sets interior to a rectangle $R$, then $h_{0}$ can be extended to an area-preserving automorphism of the plane equal to the identity outside of $R$.

Proof. Let $H_{0}$ denote the set of automorphisms of the plane equal to the identity outside of $R$. By a theorem of Kuratowski [3, vol. II, p. 383] (essentially due to Antoine) $h_{0}$ can be extended to an automorphism $h_{1}$ of the plane, and we can take $h_{1} \in H_{0}$. Let $L$ be a closed line segment interior to $R$. By the same theorem, there exists an automorphism $h_{2} \in H_{0}$ such that $h_{2}(L) \supset K_{1}$, and by modifying $h_{2}$ appropriately on the segments of $L$ outside $h_{2}^{-1}\left(K_{1}\right)$ we can insure that $\mu\left(h_{2}(L)\right)=\mu\left(K_{1}\right)$ and $\mu\left(h_{1} h_{2}(L)\right)=\mu\left(K_{2}\right)$ (cf. [6, p. 902, Lemma 17]). Then the measures defined in $R$ by $\mu_{1}(A)=\mu\left(h_{2}(A-L)\right)$ and $\mu_{2}(A)$ $=\mu\left(h_{1} h_{2}(A-L)\right)$ are topologically equivalent [6, p. 887, Corollary 1]. Moreover, since $\mu_{1}(L)=\mu_{2}(L)=0$ there exists an automorphism $g$ equal to the identity on $L$ as well as outside $R$, such that $\mu_{2}(g(A))=\mu_{1}(A)$ for all Borel sets [6, p. 888, Corollary 4]. It follows that the transformation $h=h_{1} h_{2} g h_{2}^{-1}$ is an area-preserving automorphism of the plane, equal to the identity outside $R$ and equal to $h_{0}$ on $K_{1}$.

REMARK 1. It would be desirable to have a more direct proof of Theorem 6. In some cases this is easy. For instance, if $E$ is a set of the form $E=\{(x, y): y-F(x) \in B\}, F$ measurable, $m(B)>0$, then by Lusin's theorem there exists a continuous function $G(x)$ equal to $F(x)$ on a set $A$ of positive measure, and the equations $x^{\prime}=x, y^{\prime}=y-G(x)$ define an area-preserving automorphism $h$ such that $h(E) \supset A \times B$.

REMARK 2. It is an open question whether there exists an (M)-partition of the plane that retains the property under all 1:1 transformations of class 
$C^{\prime}$, or under all automorphisms that satisfy a Lipschitz condition.

5. A question of category. In this section we return to the original problem, that of constructing an (M)-partition of the unit square into two sets, and ask whether the property of being a member of such a partition is exceptional or not among measurable subsets of the square. Let $S$ be the complete metric space consisting of all measurable subsets $E$ of the unit square $Q$, equivalent sets being identified, with the usual metric: $\rho(E, F)=\mu(E \ominus F)$. If a set $E \subset Q$ and its complement $E^{\prime}=Q-E$ both have property (M) relative to $Q$ then so does every set equivalent to $E$, hence the sets that are members of some (M)-partition of $Q$ constitute a certain subset $M$ of $S$.

THEOREM 7. $S-M$ is of first category in $S$.

Proof. Let $P$ denote the set of all $E \in S$ such that for some pair $(A, B)$ of linearly measurable subsets of the unit interval, $\mu((A \times B)-E)=0$ and $m(A) m(B)>0$. As in $\S 1, m(A) m(B)=\int m(A \cap(x+B)) d x$ and $m(A \cap(x+B))$ $>0$ on some subinterval of $[-1,1]$. Let $\left\{I_{n}\right\}$ be an enumeration of all subintervals of $[-1,1]$ with rational end points, and let $F_{n}$ be the class of ordered pairs $(A, B)$ such that $m(A \cap(x+B))>1 / n$ on $I_{n}$. Then each pair $(A, B)$ with $m(A) m(B)>0$ belongs to at least one class $F_{n}$. Hence if we denote by $P_{n}$ the set of all $E \in S$ such that $\mu((A \times B)-E)=0$ for at least one pair $(A, B)$ belonging to $F_{n}$, then $P=\bigcup_{1}^{\infty} P_{n}$. To show that $P$ is of first category it will therefore suffice to show that $P_{n}$ is nowhere dense.

Let $n>0, E_{0} \in S$, and $\epsilon>0$ be given. Choose a subinterval $J$ of $I_{n}$ with $m(J)<\epsilon$ and let $E_{J}$ consist of all points $(x, y)$ of the unit square such that $x-y \in J$. Put $E_{1}=E_{0}-E_{J}$, then $\rho\left(E_{1}, E_{0}\right) \leqq \mu\left(E_{J}\right)<\epsilon$. Take $\delta=m(J) / n$ and consider any $E \in S$ with $\rho\left(E, E_{1}\right)<\delta$. For any pair $(A, B)$ in $F_{n}$ we have

$$
((A \times B)-E) \cup\left(E-E_{1}\right) \supset(A \times B)-E_{1} \supset\left((A \times B) \cap E_{J}\right),
$$

hence

$$
\begin{aligned}
\mu((A \times B)-E)+\mu\left(E-E_{1}\right) & \geqq \mu\left((A \times B) \cap E_{J}\right) \\
& =\iint \chi_{J}(x-y) \chi_{A}(x) \chi_{B}(y) d x d y \\
& =\int_{J} m(A \cap(x+B)) d x \geqq \frac{m(J)}{n}=\delta .
\end{aligned}
$$

Therefore $\mu((A \times B)-E) \geqq \delta-\rho\left(E, E_{1}\right)>0$, and so $E \notin P_{n}$. This shows that $P_{n}$ is nowhere dense in $S$; in fact, within any sphere in $S$ of radius $2 \epsilon$ we have shown that there is a sphere of radius $\delta$ that contains no point of $P_{n}$.

The mapping $T: T E=E^{\prime}$ (complementation) is an isometry of $S$. Hence $P \cup T P$ is likewise of first category. Since $P \cup T P=S-M$, it follows that $M$ is a residual set in $S$.

6. Partitions of general product spaces. Turning now to consideration of 
the product of two general measure spaces, we first show that (M)-partitions are preserved under isomorphism of the component spaces. Two totally $\sigma$-finite measure spaces are said to be isomorphic if there exists a measurepreserving isomorphism between their measure algebras.

Lemma B. If $(X, \mu)$ and $(Y, \nu)$ are totally $\sigma$-finite measure spaces isomorphic respectively to $\left(X^{\prime}, \mu^{\prime}\right)$ and $\left(Y^{\prime}, \nu^{\prime}\right)$, then the product spaces $(X \times Y, \mu \times \nu)$ and $\left(X^{\prime} \times Y^{\prime}, \mu^{\prime} \times \nu^{\prime}\right)$ are isomorphic under a correspondence that makes measurable product sets correspond to measurable product sets. Thereby any (M)-partition of $X \times Y$ corresponds to an (M)-partition of $X^{\prime} \times Y^{\prime}$.

Proof. We may assume that $(X, \mu)$ and $(Y, \nu)$ are finite measure spaces, since the general case can be reduced to this by a direct sum decomposition of each. Then any isomorphism between the measure algebras of $(X, \mu)$ and $\left(X^{\prime}, \mu^{\prime}\right)$ defines a correspondence between the idempotents (characteristic functions) of $L_{2}(\mu)$ and $L_{2}\left(\mu^{\prime}\right)$ which can be extended uniquely to a unitary transformation $f \rightarrow f^{\prime}$ of $L_{2}(\mu)$ onto $L_{2}\left(\mu^{\prime}\right)$ which is multiplicative, that is, such that $f_{1} f_{2}=f_{3}$ a.e. implies $f_{1}^{\prime} f_{2}^{\prime}=f_{3}^{\prime}$ a.e. [7, Theorem 4.4]. Similarly, let $g \rightarrow g^{\prime}$ be such a mapping of $L_{2}(\nu)$ onto $L_{2}\left(\nu^{\prime}\right)$. Then the correspondence

$$
\sum_{i=1}^{n} c_{i} f_{i}(x) g_{i}(y) \rightarrow \sum_{i=1}^{n} c_{i} f_{i}^{\prime}\left(x^{\prime}\right) g_{i}^{\prime}\left(y^{\prime}\right)
$$

defines a linear isometric transformation from a dense subset of $L_{2}(\mu \times \nu)$ onto a dense subset of $L_{2}\left(\mu^{\prime} \times \nu^{\prime}\right)$, and by continuity this can be extended to a unitary transformation $S$ of $L_{2}(\mu \times \nu)$ onto $L_{2}\left(\mu^{\prime} \times \nu^{\prime}\right) . S$ is likewise multiplicative, hence idempotents correspond to idempotents. The restriction of $S$ to these defines an isomorphism between the measure algebras of $(X \times Y, \mu \times \nu)$ and $\left(X^{\prime} \times Y^{\prime}, \mu^{\prime} \times \nu^{\prime}\right)$ under which product sets correspond to product sets. Consequently an (M)-partition of either product space determines an (M)partition of the other, modulo nullsets.

THEOREM 8. The product of two totally $\sigma$-finite measure spaces $(X, \mu)$ and $(Y, \nu)$ admits an $(\mathrm{M})$-partition if and only if the measure algebras of the given spaces are nonatomic.

Proof. Suppose the measure algebra of $(X, \mu)$ contains an atom. Replacing $(X, \mu)$ by an isomorphic space, we can assume that for some point $x_{0} \in X$ the set $A=\left\{x_{0}\right\}$ has positive measure. Let $X \times Y=E_{1} \cup E_{2}$ be an arbitrary partition of $X \times Y$ into measurable sets, and put $B=\left\{y:\left(x_{0}, y\right) \in E_{1}\right\}$. If $\nu(B)=0$ then $(\mu \times \nu)\left(E_{1} \cap(A \times Y)\right)=0$. If $\nu(B)>0$ then $(\mu \times \nu)(A \times B)>0$ and $(\mu \times \nu)$ $\left(E_{2} \cap(A \times B)\right)=0$. Thus property (M) fails for $E_{1}$ or $E_{2}$; hence no (M)-partition of $X \times Y$ is possible. Similar considerations apply in case $(Y, \nu)$ contains an atom. The condition is therefore necessary.

To prove sufficiency, first decompose $X$ and $Y$ into finite measure spaces. By a theorem of Maharam [4], any finite nonatomic measure algebra is a 
direct sum of countably many homogeneous measure algebras, and (after normalization) any homogeneous measure algebra can be represented as a product of suitably many replicas of the Lebesgue measure space on the unit interval. Hence, after appropriate decomposition of the component spaces, it only remains to prove the theorem for the case in which both $(X, \mu)$ and $(Y, \nu)$ are products of families of intervals with Lebesgue measure $m$. Select an interval $X_{0}$ and $Y_{0}$ from each of these families and write $X=X_{0} \times X_{1}$, $Y=Y_{0} \times Y_{1}$. Let $\left\{E_{i}^{\prime}\right\}$ be an (M)-partition of the square $X_{0} \times Y_{0}$, and let $E_{i}$ be the cylinder set in $X \times Y$ based on $E_{i}^{\prime}$. To show that $\left\{E_{i}\right\}$ is an (M)partition of $X \times Y$, consider any non-null measurable sets $A \subset X$ and $B \subset Y$. Then $0<\mu(A)=\int d x_{0} \int \chi_{A}\left(x_{0}, x_{1}\right) d x_{1}$, hence there exists a non-null set $A_{0} \subset X_{0}$ and a positive number $p$ such that $\int \chi_{A}\left(x_{0}, x_{1}\right) d x_{1} \geqq p \chi_{A_{0}}\left(x_{0}\right)$. Similarly there exists a non-null set $B_{0} \subset Y_{0}$ and a positive number $q$ such that $\int \chi_{B}\left(y_{0}, y_{1}\right) d y_{1}$ $\geqq q \chi_{B_{0}}\left(y_{0}\right)$. Consequently,

$$
\begin{aligned}
(\mu \times \nu)\left(E_{\boldsymbol{i}} \cap(A \times B)\right) & =\iint \chi_{E_{i}^{\prime}}\left(x_{0}, y_{0}\right) d x_{0} d y_{0} \int \chi_{A}\left(x_{0}, x_{1}\right) d x_{1} \int \chi_{B}\left(y_{0}, y_{1}\right) d y_{1} \\
& \geqq p q \iint \chi_{E_{i}^{\prime}}\left(x_{0}, y_{0}\right) \chi_{A_{0}}\left(x_{0}\right) \chi_{B_{0}}\left(y_{0}\right) d x_{0} d y_{0} \\
& =p q(m \times m)\left(E_{i}^{\prime} \cap\left(A_{0} \times B_{0}\right)\right)>0 .
\end{aligned}
$$

As an application, consider any two nonatomic measure spaces $(X, \mu)$ and $(Y, \nu)$ with $\mu(X)=\nu(Y)=1$. Let $R$ denote the ring of all finite unions of measurable product sets in $X \times Y$, and let $S$ denote the $\sigma$-ring of measurable subsets of $X \times Y$. Between $R$ and $S$ there is another ring of some interest, namely the ring $R_{1}$ of all sets with equal outer and inner content, the outer (inner) content of any set $E \subset X \times Y$ being defined as the lower (upper) bound of $\mu \times \nu$ on all supersets (subsets) of $E$ that belong to $R$. Then $\mu \times \nu$ on $R_{1}$ is the least complete extension of $\mu \times \nu$ on $R$ as a content [5]. In general $R_{1}$ contains $R$ as a proper subring; for instance in the case of the square, $R_{1}$ includes all Jordan measurable subsets as well as all measurable product sets. The existence of an (M)-partition of $X \times Y$ implies that there are sets in $S$ with the property that every equivalent set has inner content zero and outer content one. Consequently $R_{1}$ is a proper subclass of $S$, even neglecting sets of measure zero. It follows that under present assumptions the Boolean algebra of $R_{1}$ modulo sets of content zero is never a $\sigma$-algebra and, considered as a metric space, it is always incomplete.

As a second application of Theorem 8 , let $(X, \mu)$ and $(Y, \nu)$ be two replicas of the Kakutani space [1] corresponding to Lebesgue measure on the unit interval. That is, $X=Y=$ the compact, totally disconnected, Hausdorff space determined by the Stone representation of the Lebesgue measure algebra, and $\mu=\nu=$ the completion of the measure defined on the closed open subsets of $X$ by the representation. Then the nullsets of $\mu$ are the nowhere dense sub- 
sets of $X$, and the domain of $\mu$ consists of all sets of the form $A \ominus N$, where $A$ is closed open and $N$ is nowhere dense. Consequently every non-null measurable product set in $X \times Y$ contains a base neighborhood, and so a subset of $X \times Y$ has property (M) if and only if it is metrically dense in $X \times Y$. The (M)-partitions of this product space are therefore identical with its partitions into metrically dense sets. The existence of such a partition implies that $(X \times Y, \mu \times \nu)$ is not a Kakutani space, since a Kakutani space does not admit a partition into even two dense measurable sets. Nevertheless, by Lemma $\mathrm{B},(X \times Y, \mu \times \nu)$ is isomorphic to Lebesgue measure on the unit square, and therefore isomorphic to $(X, \mu)$ itself.

\section{Bibliography}

1. P. R. Halmos, On a theorem of Dieudonne, Proc. Nat. Acad. Sci. U.S.A. vol. 35 (1949) pp. $38-42$.

2. H. Helson, Remark on measures in almost-independent fields, Studia Mathematica vol. 10 (1948) pp. 182-183.

3. C. Kuratowski, Topologie I, 2d ed., Warszawa-Wrocław, 1948, and Topologie II, 1950.

4. D. Maharam, On homogeneous measure algebras, Proc. Nat. Acad. Sci. U.S.A. vol. 28 (1942) pp. 108-111.

5. K. Mayrhofer, Inhalt und Mass, Vienna, 1952.

6. J. C. Oxtoby and S. M. Ulam, Measure-preserving homeomorphisms and metrical transitivity, Ann. of Math. vol. 42 (1941) pp. 874-920.

7. I. E. Segal, Equivalences of measure spaces, Amer. J. Math. vol. 73 (1951) pp. 275-313.

8. H. Steinhaus, Sur les distances des points des ensembles de mesure positive, Fund. Math. vol. 1 (1920) pp. 93-104.

9. A. Weil, L'intégration dans les groupes topologiques et ses applications, Actualités Scientifiques et Industrielles, no. 869, Paris, 1940.

The University of Notre Dame,

Notre DAME, IND.

Bryn Mawr College,

Bryn MaWr, Pa. 